\title{
Determination of fundamental parameters and circumstellar properties for a sample of $B[e]$ stars
}

\author{
María F. Muratore ${ }^{1}$, Lydia S. Cidale ${ }^{1,2}$, María L. Arias ${ }^{1,2}$, \\ Juan Zorec ${ }^{3}$ and Andrea F. Torres ${ }^{1,2}$ \\ ${ }^{1}$ Facultad de Ciencias Astronómicas y Geofísicas, Universidad Nacional de La Plata, \\ Paseo del Bosque S/N, 1900 La Plata, Argentina \\ email: fmuratore@carina.fcaglp.unlp.edu.ar \\ ${ }^{2}$ Instituto de Astrofísica La Plata, CCT La Plata, CONICET, \\ Paseo del Bosque S/N, 1900 La Plata, Argentina \\ ${ }^{3}$ Institut d'Astrophysique de Paris, UPMC, UMR7095 CNRS, Paris, France
}

\begin{abstract}
We develop a simple model to derive theoretical continuum energy distributions for $\mathrm{B}[\mathrm{e}]$ stars, consisting of a B star surrounded by an envelope made of gas and dust. We select a sample of $\mathrm{B}[\mathrm{e}]$ objects for which we construct the observed energy distributions, from 0.1 to $100 \mu \mathrm{m}$, using available photometric and spectroscopic data. We present some preliminary fittings.
\end{abstract}

Keywords. stars: emission-line, Be, circumstellar matter, stars: fundamental parameters

\section{Introduction}

Stars with the $\mathrm{B}[\mathrm{e}]$ phenomenon are mainly characterized by the presence of forbidden emission lines in the visual wavelength range and strong IR dust emission. This phenomenon is observed in stars in different evolutionary phases (Lamers et al. 1998) and many of these objects have "unclear" evolutionary states. In most cases, the determination of the star's properties is rather uncertain, as the circumstellar envelopes frequently blur the photospheric characteristics. Furthermore, the interstellar extinction is difficult to estimate and the unknown or inaccurate distances lead to uncertain luminosities. In this work we present a simple model of an envelope made of gas and dust and analyze its effect on the spectral energy distribution (SED) of a normal B star. Comparing theoretical results with observations we aim at deriving fundamental parameters of the star as well as global physical properties of the gas and dust envelopes.

\section{The envelope model}

We consider the circumstellar material distributed in a gaseous region located close to the $\operatorname{star}\left(\approx 3 R_{*}\right)$ and a dusty region, far from the star $\left(\gtrsim 100 R_{*}\right)$.

Circumstellar gas: The proposed model of the gaseous circumstellar envelope is based on those presented in Cidale \& Ringuelet (1989), Moujtahid (1998) and Moujtahid et al. (1999). We calculate the emergent flux of a system formed by a star + spherical envelope by considering that the envelope can be reduced to an equivalent shell. We can then apply a plane-parallel solution for the transfer equation. The observed flux at a distance $D$ obtained in this way can be expressed as:

$$
f_{\lambda}^{*+G}=\frac{R_{*}^{2}}{D^{2}} F_{\lambda}^{*} \alpha_{\lambda}\left(\frac{R_{*}}{R_{G}}, \tau_{\lambda}^{G}\right)+\frac{R_{G}^{2}}{D^{2}} S_{\lambda}\left(T_{G}\right) \beta_{\lambda}\left(\frac{R_{*}}{R_{G}}, \tau_{\lambda}^{G}\right)
$$



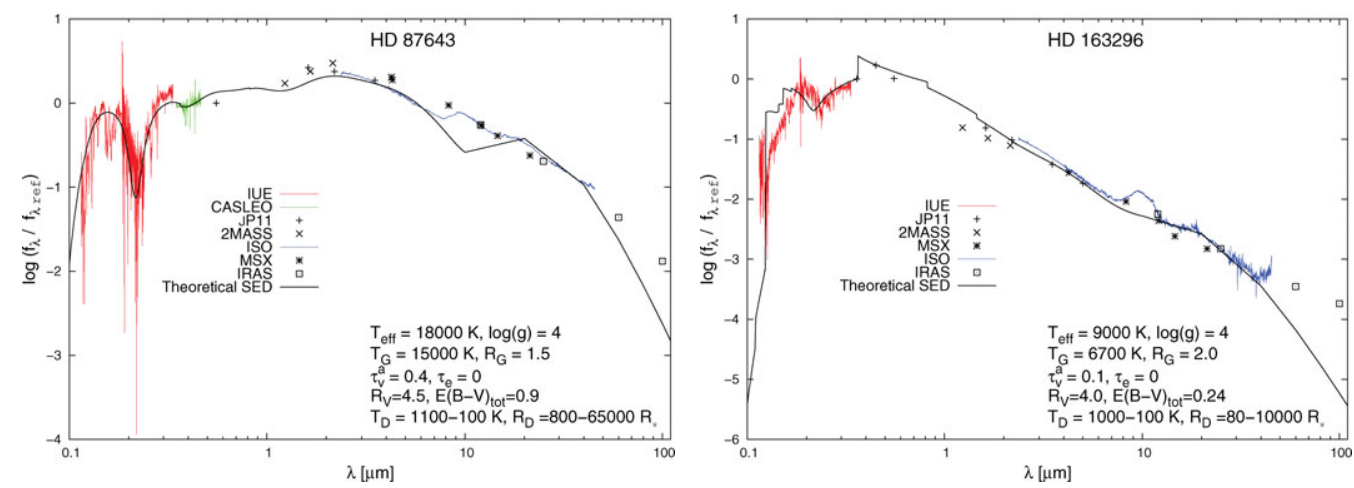

Figure 1. Theoretical and observed energy distributions for two $\mathrm{B}[\mathrm{e}]$ stars

with $\tau_{\lambda}^{G}$, optical depth of the gaseous shell; $R_{*}$, stellar radius; $R_{G}$, gaseous shell effective radius; $F_{\lambda}^{*}$, photospheric stellar flux; $S_{\lambda}\left(T_{G}\right)$, source function; $T_{G}$, electron temperature of the gas, $\alpha_{\lambda}$ and $\beta_{\lambda}$, functions that can be numerically calculated.

Circumstellar and Interstellar dust: The circumstellar dust region is treated using the same scheme proposed for the gaseous region. We characterize the dust by a total optical depth $\tau_{\lambda}^{D}$, a temperature $T_{D}$ and a source function $S_{\lambda}=B_{\lambda}\left(T_{D}\right)$. We represent the interstellar extinction by $\tau_{\lambda}^{I S M}$. Both, $\tau_{\lambda}^{D}$ and $\tau_{\lambda}^{I S M}$ are related to the absorption $A(\lambda)$ through the expression: $\tau=0.4 \ln (10) A(\lambda)$. Using the law given by Cardelli, Clayton \& Mathis (1989): $A(\lambda)=\left[R_{v} a(1 / \lambda)+b(1 / \lambda)\right] E(B-V)$, where $R_{v}$ is the total to selective extinction and $E(B-V)$ is the color excess. We adopt $R_{v}^{I S M}=3.1$ for the interstellar dust, while for the circumstellar dust we try different values of $R_{v}^{D}$ greater than 3.1.

Observed flux: Finally, we obtain an expression for the observed flux at a distance $D$, for: star + gaseous shell + dust shell + interstellar extinction given by:

$$
f_{\lambda}^{*+G+D+I S M}=f_{\lambda}^{*+G} e^{-\tau_{\lambda}^{D}}+\frac{R_{D}^{2}}{D^{2}} S_{\lambda}\left(T_{D}\right)\left[1-2 E_{3}\left(2 \tau_{\lambda}^{D}\right)\right] e^{-\tau_{\lambda}^{I S M}}
$$

In order to eliminate the dependence on the distance $D$, we normalize the flux to that at a reference wavelength $\lambda_{\text {ref }}$. Using expression (2.2) we obtain the theoretical SED, from 0.1 to $100 \mu \mathrm{m}$, for different sets of the free parameters of our model: $R_{G}, T_{G}, \tau_{\lambda}^{G}, R_{D}$, $T_{D}, R_{v}^{D}, E_{D}(B-V)$ and $E_{I S M}(B-V)$.

\section{Results}

Photometric and spectroscopic data for $\mathrm{B}[\mathrm{e}]$ stars is scarce and/or cover small wavelength ranges. We gathered all the information found in the literature and were able to build observed SEDs that cover a great percentage of the modeled range. In the following figures we present the observed SED together with the best theoretical fit for two objects of our sample: HD87643 and HD163296.

\section{References}

Cardelli, J. A., Clayton, G. C., \& Mathis, J. S. 1989, ApJ, 345, 245

Cidale, L. S. \& Ringuelet, A. E. 1989, PASP, 101, 417

Lamers, H. J. G. L. M., Zickgraf, F.-J., de Winter, D., Houziaux, L. et al. 1998, A\&A, 340, 117

Moujtahid A. 1998, Thèse de Doctorat, Université Paris VI

Moujtahid, A., Zorec, J., \& Hubert, A. M. 1999, A\&̛A, 349, 151 